\title{
Searches for electroweak production of supersymmetric gaugi- nos and sleptons with the ATLAS detector
}

\author{
Huan Ren - on behalf of ATLAS collaboration ${ }^{1, a}$ \\ ${ }^{1}$ Institute of High Energy Physiscs, Beijing, China
}

\begin{abstract}
This paper shows the summary of the results from some recent research by ATLAS SUSY electroweak group(EWK). The sub-topics are including direct stau production, same-sign chargino pair production via vector boson fusion(VBF) and compressed spectra in direct production.
\end{abstract}

\section{Introduction}

Since the discovery of Higgs boson at LHC, the most essential part of standard model(SM) was confirmed. However, we still have some remaining issues after this: hierachy problem, dark matter, origin of gravity and no gauge unification at higher scale. Supersymmetry(SUSY) has become a very appealing extension of SM to answer these questions. Currently, we have set limits of most SUSY models probe masses up to $900 \mathrm{GeV}$ (squarks) and $1.4 \mathrm{TeV}$ (gluinos).

The cross-sections of different SUSY procedure are shown on Fig. 1. The EWK sector provides a promising approach for new physics among all SUSY searches. The hadronic activity is quite low in a certain EWK process despite the low production cross-section. For most of the EWK models, we have 1-4 leptons in the final state, as well as large missing transverse energy(Etmiss) and 0-2 jets(or b-jets).

\section{Search for direct stau production in events with at least two hadronic taus and missing transverse momentum using multivariate analysis technique}

This part is an update of JHEP 1410(2014)96.

\section{1 direct stau production}

The direct stau production(see Fig. 2) have the final states: two opposite-sign hadronically decaying taus, no jet, and large $E_{\text {miss }}^{T}$. Limited sensitivity was observed using cut-and-count analysis due to the low cross-section of direct stau process. Multivariate analysis(MVA) is used to improve this result. We defined one boosted decision tree(BDT) signal region(SR) for this intension, as shown on Fig. 3.

ae-mail: huan.ren@cern.ch 


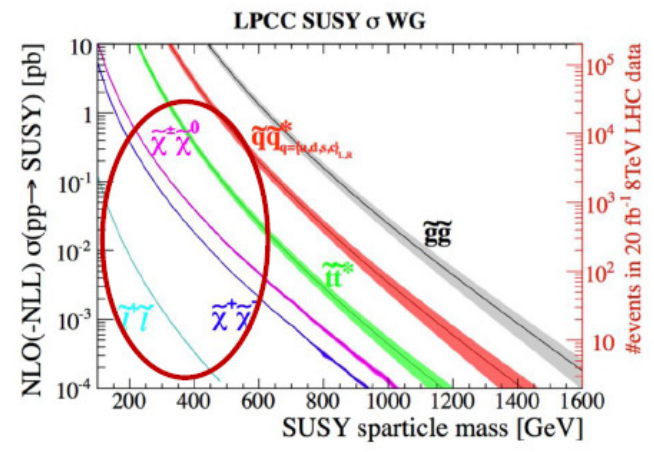

Figure 1. SUSY cross-sections for different production against SUSY particle mass.

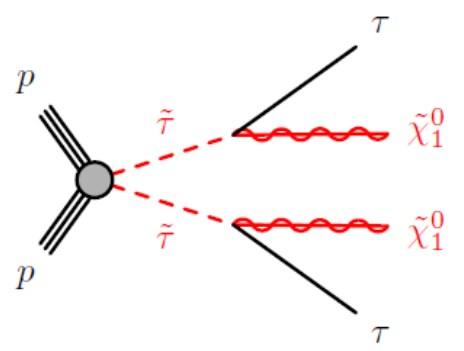

Figure 2. Feynman diagram of the pair production of charged staus decaying into 2 taus in the final state.

\section{2 background}

The main background of this study includes:

- $\mathrm{W}+\mathrm{jets}(1$ real +1 fake lepton). This background is estimated by a normalization to data in dedicated WCR.

- multi-jet. We apply an "ABCD" estimation to this background source, using tau-ID and $m_{\mathrm{T} 2}$ as the two uncorrelated variables, shown in Fig. 4.

- Other sub-dominant background. This is estimated by Monte Carlo(MC) simulation.

\section{3 results}

The results of this direct stau update was shown in Fig. 5. Significant improvement is observed in the low neutralino mass region using MVA compared to cut-and-count analysis. 


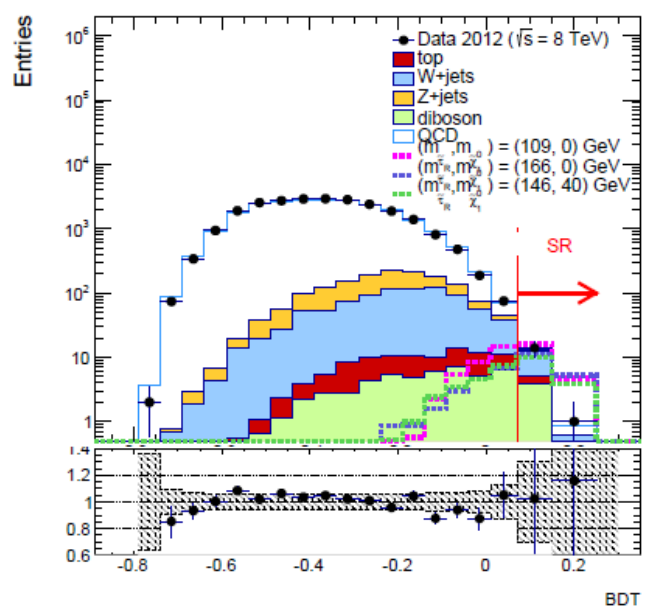

Figure 3. Distribution of BDT response and the SR definition.

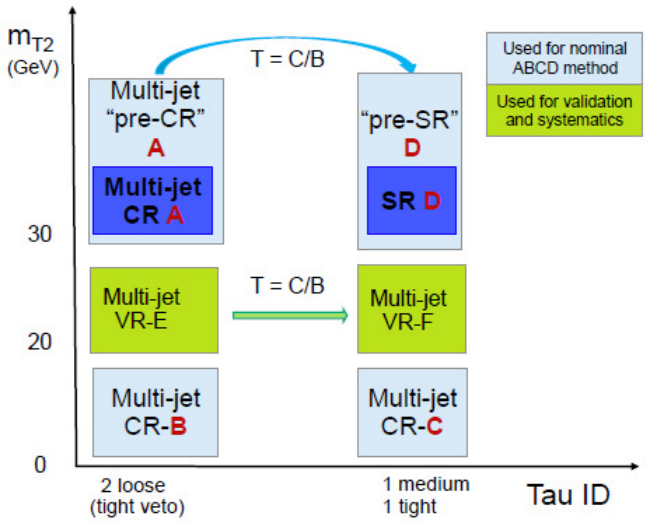

Figure 4. Introduction of "ABCD" estimation. Two uncorrelated variables are used on each axis, defining 3 CRs with enough statistics and $1 \mathrm{SR}$. The extrapolation from the CRs provides a good estimation to the event yields in SR.

\section{Search for supersymmetry in compressed scenarios with two and three leptons and missing transverse momentum in the final state}

\subsection{Same-sign $\mathrm{C} 1 \mathrm{C} 1$}

The same-sign(SS) C1C1(two chargino-one mediate process) production(see Fig. 6) have a final state with: two SS light leptons, $\geq 2$ jet and large $E_{\text {miss }}^{T}$. 

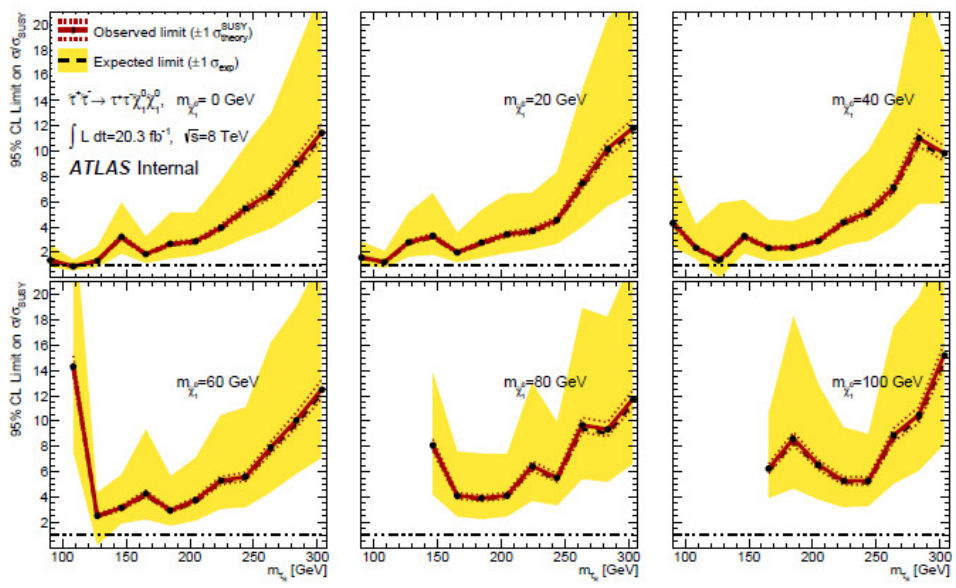

Figure 5. Upper limits on the signal strength for production of left and right-handed stau pairs for different neutralino masses.

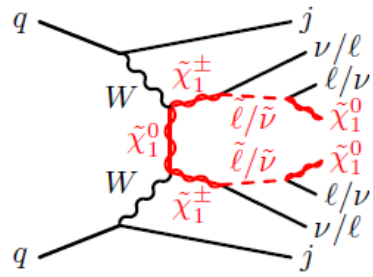

Figure 6. Feynman diagram for VBF production of $\mathrm{C} 1 \mathrm{C} 1$ with two SS leptons in the final states.

\section{2 background}

The SR of this analysis includes the following main background:

- "prompt" leptons(diboson, H), estimated from MC simulation directly.

- non-prompt(fake) leptons(W+jets, $t \bar{t})$ - Fake Factor Method was adopted for the estimation.

- Charge-misID leptons - measured from dedicated control region(CR).

The disctributions of $E_{m i s s}^{T}$ and $\mathrm{Mjj}$ are shown on Fig. 7.

\section{3 results}

The fitting results of of this topic was shown on Fig.8. The best observed upper limit is found for $\widetilde{\chi}_{1}^{0}$ mass of $120 \mathrm{GeV}$ and $\mathrm{m}\left(\widetilde{\chi}_{1}^{ \pm}\right)-\mathrm{m}\left(\widetilde{\chi}_{1}^{0}\right)=25 \mathrm{GeV}$. Slightly stronger sensitivity is also observed for higher $\tilde{\chi}_{1}^{ \pm}$masses.

\section{Search for supersymmetry in compressed scenarios}

The compressed scenarios are some SUSY scenarios with small mass difference between sparticles and neutralinos, leading to low-momentum leptons in final states. Generally, low mass-splitting, high 

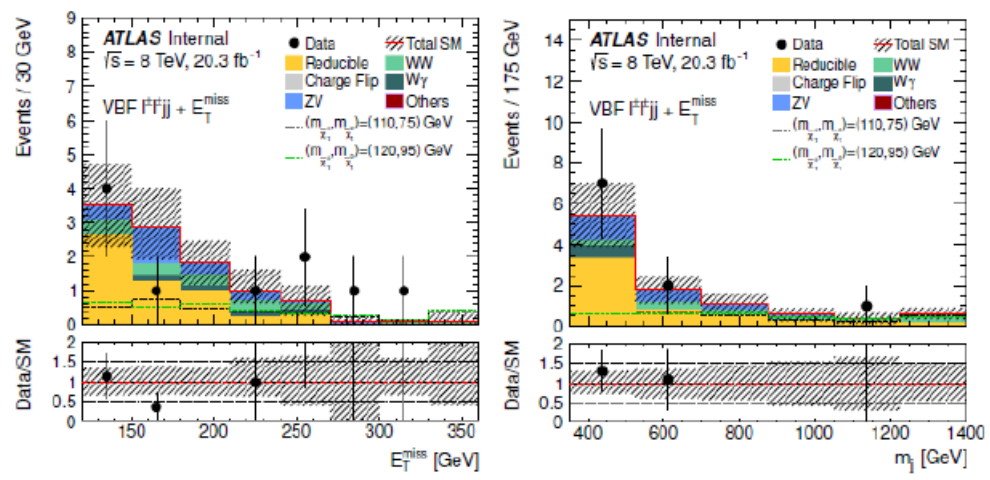

Figure 7. Distribution of the $E_{\text {miss }}^{T}$ and di-jet invariant mass in SR.
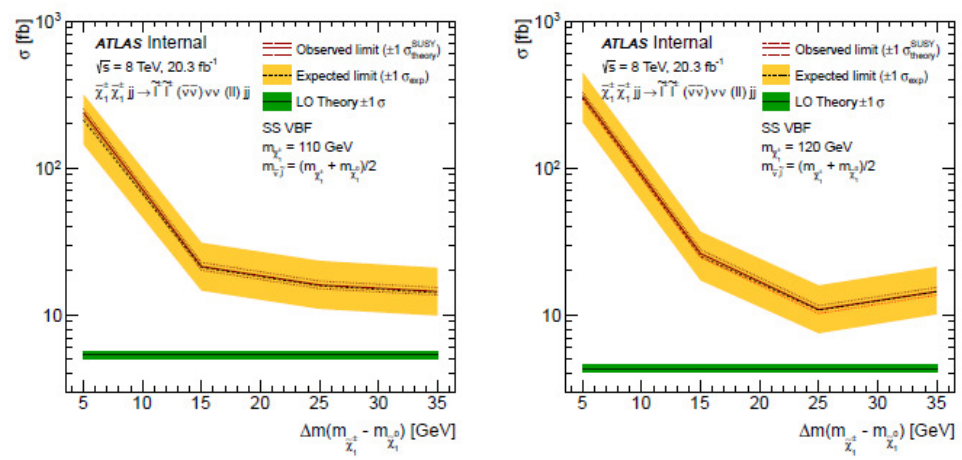

Figure 8. 95\% CL exclusion limits on the cross-section for VBF production of $\widetilde{\chi}_{1}^{ \pm} \widetilde{\chi}_{1}^{ \pm}$. The limits have been set with respect to the mass difference between the $\widetilde{\chi}_{1}^{ \pm}$and $\widetilde{\chi}_{1}^{0}$.

soft decay products and SM-like behaviour leads to many difficulties for this analysis. The results of re-optimization of three sub-topics are shown:

- two opposite-sign(OS) light leptons.

- two same-sign(SS) light lrptons.

- three light leptons.

\subsection{Two OS light leptons: Event selection and background estimation}

The experimental signature of this study is: two OS light leptons and $E_{m i s s}^{T}$ shown in Fig. 9. Two SRs are defined for this analysis, requiring a high pT initial-state-radiation(ISR) jets, which are sensitive to small and moderate mass splittings. Discriminate variabls "Super razor" are also used in the SR definition, shown on Fig. 10. The main background in the SRs are:

- Irreducibel background(WW, top, ZV), estimated by normalized MC simulation in dedicated CRs.

- Reducibel background(all fake sources) - Matrix Method adopted for this background source. 
- Others(Higgs, Z+jets) - measured by MC simulation directly.

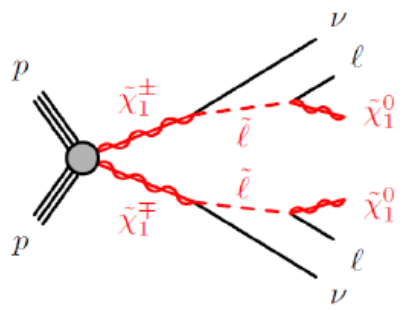

Figure 9. Feynman diagram for compressed scenario with two OS leptons in the final states.

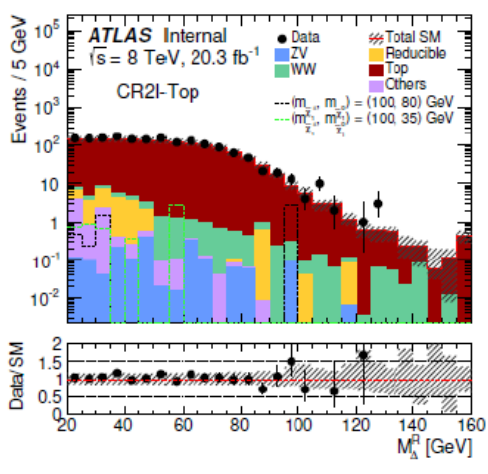

(a) $M_{\Lambda}^{R}$ in the top $\mathrm{CR}$

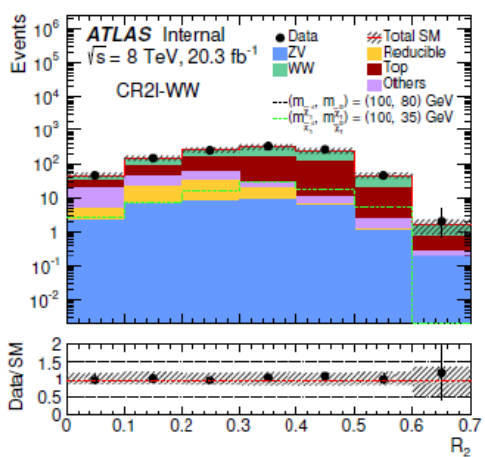

(c) $R_{2}$ in the $W W \mathrm{CR}$

Figure 10. Distributions of the "super razor" varibales in SRs.

\subsection{Two SS light leptons: Event selection and background estimation}

The experimental signature of this scenario is: two SS light leptons and $E_{\text {miss }}^{T}$ shown in Fig. 11. 8 BDTs are trained to cover four different mass splittings for $\mathrm{m}\left(\widetilde{\chi}_{1}^{ \pm}\right)-\mathrm{m}\left(\widetilde{\chi}_{1}^{0}\right)=20,35,60,100 \mathrm{GeV}$. For each mass splitting, one region requires an ISR jet, and the other applies a jet-veto, shown on Fig. 12.

The SM background in the SRs are:

- "prompt" leptons(diboson, triboson, $t \bar{t} \mathrm{~V}, \mathrm{tZ}, \mathrm{H}$ ), estimated from MC simulation.

- non-prompt(fake) leptons $(\mathrm{W}+\mathrm{jets}, t \bar{t})$ - Matrix Method for the estimation, except Wy by MC simulation.

- Charge-misID leptons - charge-misID rate measured from control region(CR).

\subsection{Three light leptons: Event selection and background estimation}

The final state of this scenario is: exactly three light leptons(1 same-flavor OS pair) and $E_{m i s s}^{T}$ shown in Fig. 13. 


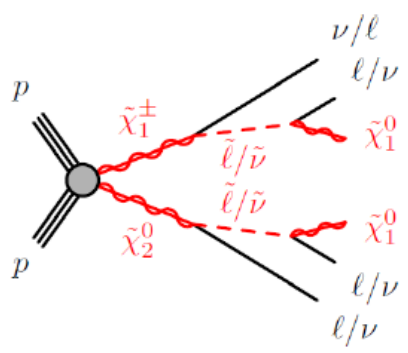

Figure 11. Feynman diagram for compressed scenario with two SS leptons in the final states.

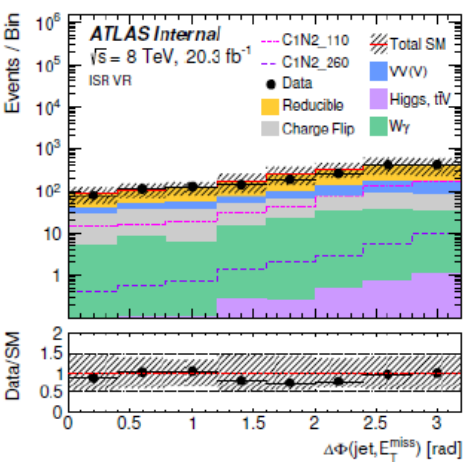

(a) $\Delta \phi$ (jet, $E_{\mathrm{T}}^{\text {miss }}$ ) in ISR VR

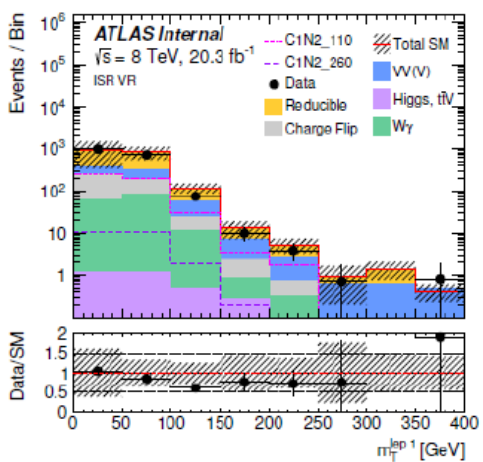

(b) $m_{\mathrm{T}}^{\text {lep1 }}$ in ISR VR

Figure 12. Distributions of $\Delta \phi$ and $m_{T}^{\text {lep } 1}$ in SRs.

Two SRs are defined with low pT leptons to target mass splittings of 4-15 and 15-25GeV, while another two SRs which request a jet with pT $>50 \mathrm{GeV}$ to target ISR events for both splitting regions.

The MET distribution in the SRs are shown on Fig. 14, with the following SM background:

- irreducible(prompt) leptons, including diboson and triboson, estimated from MC simulation.

- reducible(fake) leptons, including Vjets, WW, top and $\bar{t}$, used Matrix Method for the estimation.

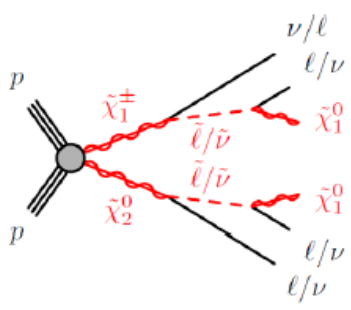

Figure 13. Feynman diagram for compressed scenario with 3 leptons in the final states. 


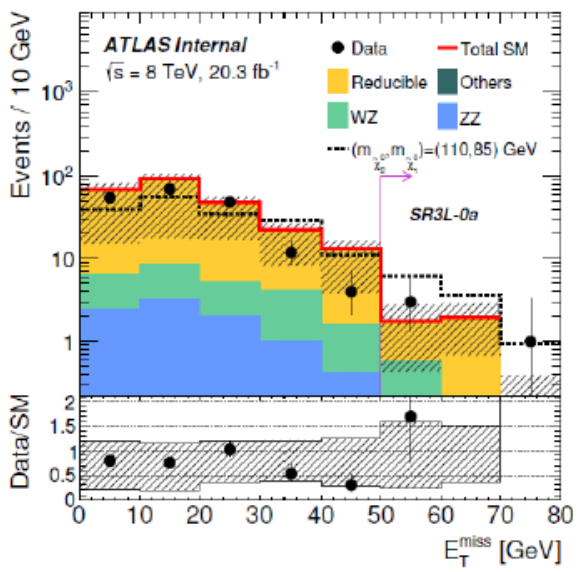

(a) $E_{\mathrm{T}}^{\mathrm{miss}}$ in SR3 $3 \ell-0 \mathrm{a}$

Figure 14. Distributions of $E_{m i s s}^{T}$ in SR.

\section{4 results}

The fitting results of all the three scenarios are shown on Fig. 15 and Fig. 16. For the OS21 analysis, reoptimized analysis nicely complements the already published one in the region of low mass splittings close to the diagonal.

For the SS21/31 analysis, re-optimized analysis nicely complements the already published one in the region of low mass splittings close to the diagonal. The combination of the new analyses give an improved sensitivity to compressed scenarios up to $\widetilde{\chi}_{1}^{ \pm}$masses of $250 \mathrm{GeV}$.

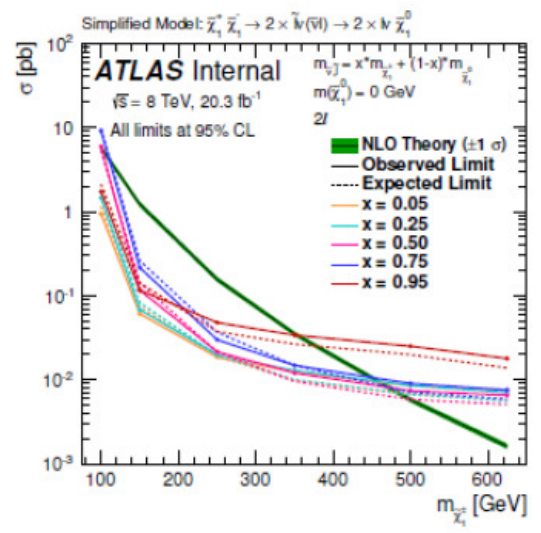

(a) Variable $\ell_{L}$ mass

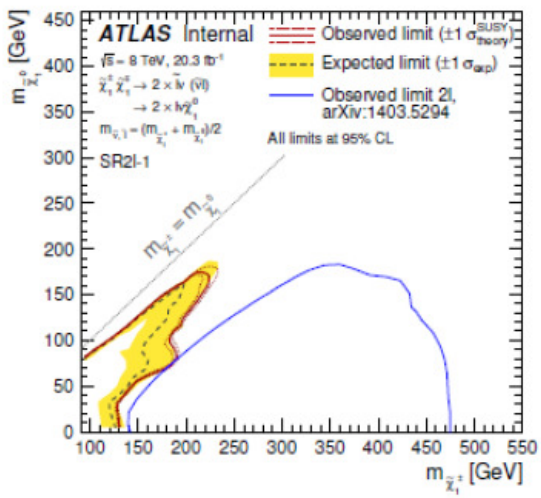

(b) $\mathrm{m}\left(\chi_{L}\right)=0.5 \times \mathrm{m}\left(\tilde{\chi}_{1}^{ \pm}\right)+0.5 \times \mathrm{m}\left(\tilde{\chi}_{1}^{0}\right)$

Figure 15. 95\% CL exclusion limits on the cross-section for compressed scenario with 2 OS leptons in the final states. 


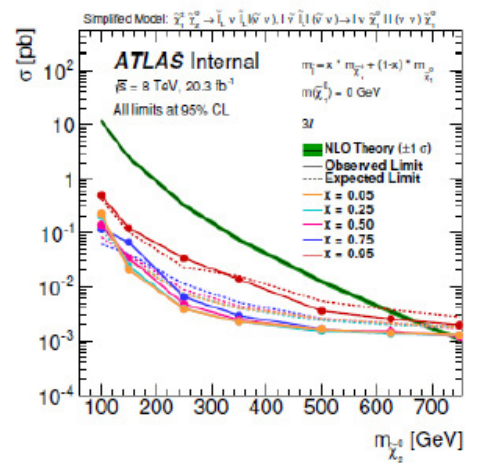

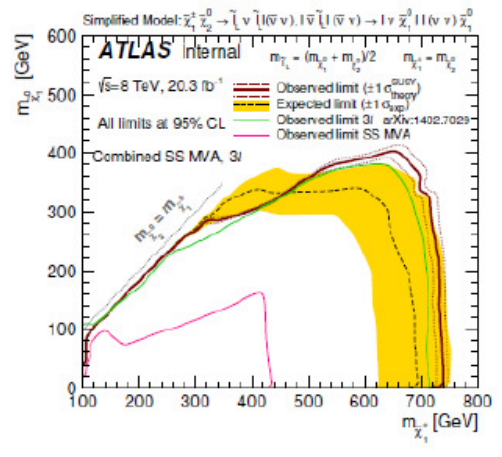

$\mathrm{m}\left(\tilde{\ell}_{L}\right)-0.5 \times \mathrm{m}\left(\tilde{X}_{1}^{ \pm}\right)+0.5 \times \mathrm{m}\left(\tilde{\chi}_{1}^{0}\right)$

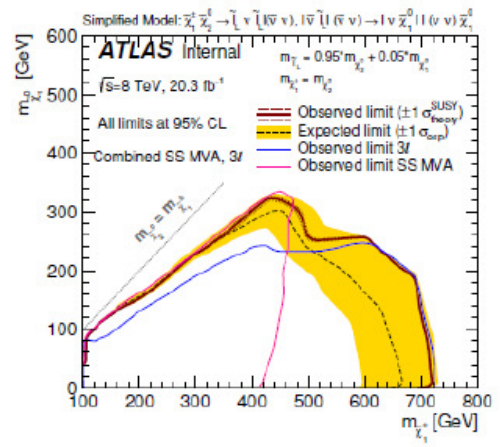

$\mathrm{m}\left(\tilde{\ell}_{L}\right)-0.95 \times \mathrm{m}\left(\tilde{\chi}_{1}^{ \pm}\right)+0.05 \times \mathrm{m}\left(\tilde{\chi}_{1}^{0}\right)$

Figure 16. 95\% CL exclusion limits on the cross-section for compressed scenario with 2 SS leptons or 3 leptons in the final states.

\section{Conclusions}

Various searches in EWK SUSY sector at ATLAS:

https://twiki.cern.ch/twiki/bin/view/AtlasPublic/SupersymmetryPublicResults

Newly updated results for EWK SUSY searching are presented, shown on Fig.17. No significant excess observed beyond SM expectation. Higher sensitivity and expanding exclusion/discovery contour is expected with 13/14TeV LHC RUN2 data.

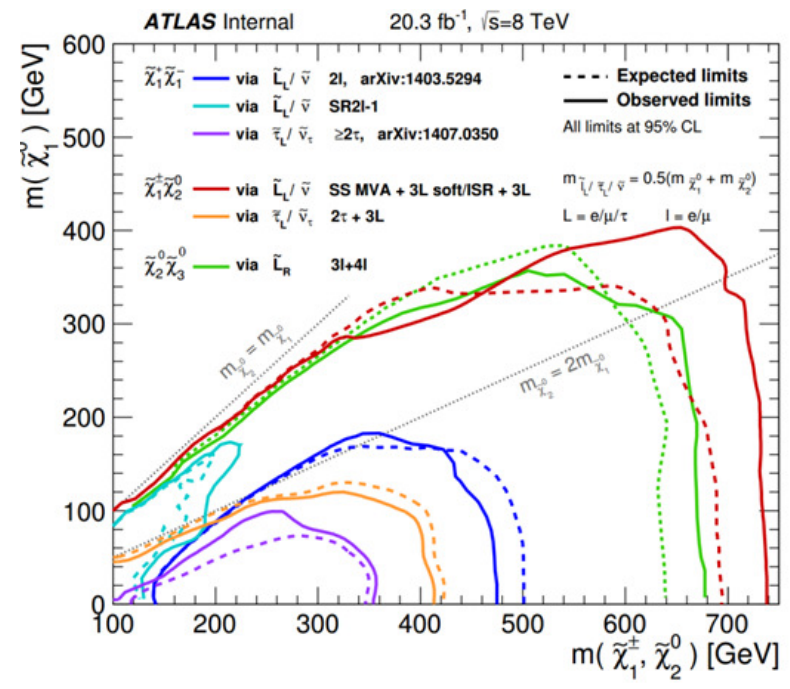

Figure 17. 95\% CL exclusion limits on the cross-section for compressed scenario with 2 SS leptons or 3 leptons in the final states. 


\section{References}

[1] ATLAS collaboration, Searches for electroweak production of supersymmetric gauginos and sleptons with the ATLAS detector, JHEP 1410(2014)96

[2] ATLAS collaboration, EWK summary paper, ATLAS-SUSY-2014-05 